\title{
Rapid extraction of machined surface data through inverse geometrical solution of tool path information
}

\author{
Lutfi Taner Tunc ${ }^{1}$
}

Received: 15 September 2015 / Accepted: 26 January 2016

(C) Springer-Verlag London 2016

\begin{abstract}
In the last decades, several process models have been developed for simulation of 5-axis milling cycles, where the simulation results are used for parameter selection or process improvement purposes. However, integrating the process models with milling cycles is not a trivial task especially for tool path modification purposes in 5-axis free-form milling. This is mainly due to the fact that tool path modification requires the machined surface information, i.e. surface location and surface normal vector, to be known. However, this information is not explicitly given in the tool path, i.e. cutter location source (CLS), file. In this paper, a novel and practical approach is proposed to analytically calculate the surface location and surface normal vectors directly from the already generated tool path in the form of CLS file. The proposed approach is applied on representative 5-axis milling cycles, and the results are verified through CAD model comparisons. It is shown that the proposed approach can calculate the machined surface data at a reasonable accuracy depending on the cutter location point density in the tool path file.
\end{abstract}

Keywords 5-Axis milling · Geometry $\cdot$ Tool path modification $\cdot$ Process simulation

\section{Introduction}

5-Axis milling is widely used in machining of free-form surfaces designed in aerospace, die-mold and automotive

Lutfi Taner Tunc

tanertunc@sabanciuniv.edu

1 Manufacturing Research Lab, Faculty of Engineering and Natural Sciences, Sabanci University, Istanbul, Turkey industries, where increased contouring capabilities are required. Ball-end mills are used to meet contouring requirement, as the tool can be oriented almost at any angle with respect to the workpiece surface, i.e. by leading and tilting the tool. Although in general, 5-axis ball-end milling increases process capability, the cutting process becomes more complicated compared to flat-end milling due to 5-axis tool motion. In other words, the engagement boundaries and the cutting conditions vary continuously along the tool axis especially at the ball-end portion of the tool. Besides, the surface properties such as surface location, surface normal and curvature vary along the tool path due to the free form of the surface.

There have been extensive process modelling effort for 5axis milling processes to simulate cutting forces [1-5], tool deflection $[6,7]$ and process dynamics $[8-10]$ for known cutting conditions. Such simulations are generally used in selection of process parameters for improved productivity. Recently, the process models have started to be integrated with tool path information to perform process simulations along milling cycles [11]. In such simulations, the tool location is obtained from the cutter location source (CLS) file and the tool envelope is used together with the rough workpiece geometry to determine engagement boundaries. Then, the simulation results can be used for tool path modification purposes such as tool path offsetting or tool axis optimization.

Tool path offsetting to compensate tool deflection is one of the mostly used tool path modification technique [12-17], where the tool location is shifted along the machined surface normal by an amount of the tool deflection. This is relatively easy in $21 / 2$ axis flat-end milling as the tool path points are co-planar and the tool axis is perpendicular or parallel to the machined surface normal so that the tool path is compensated in a plane perpendicular to the tool axis [12-15]. On the other hand, in 5-axis free-form surface milling, the tool axis is oriented with respect to the surface normal through lead and tilt 
angles, where the cutting tool trajectory, tool deflection and surface normal are in three-dimensional space [18]. Thus, either the surface location point or the surface normal vector needs to be known. However, as the surface normal vectors and surface location points are not provided in the tool path file, such information needs to be retrieved from the CLS file for automatic tool path modification so that the need for iterative tool path generation can be eliminated or minimized.

In general, tool path generation in 5-axis milling is performed in three main steps [18]. Firstly, the cutter contact (CC) points are identified by discretizing the surface to be machined into number of points according to the tolerance settings. Then, the tool axis is calculated by applying rotational transformation on the surface normal vector by amount of lead and tilt angles. Finally, the cutter location (CL) point is obtained from cutter contact points by vector addition of surface normal and tool axis multiplied by spherical radius of the tool. Throughout these steps, the required geometrical information, i.e. surface normal and cutter contact point, is obtained from the CAD model. Thus, any further tool path modification requires the surface normal vector and/or the cutter contact point need to be known, which are not explicitly given in the CLS file. As a result, the use of process simulations for tool path modification through the CLS file becomes either almost impossible or very limited. Therefore, in general, the tool path is locally modified in computer-aided manufacturing (CAM) environment.

Tool axis computation is still one of the most important challenges in 5-axis milling. Currently, CAM packages consider only the geometrical aspects of 5-axis milling, such as workpiece geometry, tool-workpiece collision and smoothness while calculating the tool axis vectors. There have been several studies on optimization of tool axis vectors based on geometrical constraints [19-23]. However, it is also important to put process mechanics and dynamics into tool axis computation, as it is shown that there may be preferable tool axis vectors for improved process mechanics and dynamics in 5-axis milling. Lim et al. [24] experimentally investigated the effect of various tool orientations in 5-axis milling of turbine blades. They applied mainly four cutting directions with lead/tilt angle combinations of $15^{\circ}$ and concluded that applying "horizontal inward with a tilt angle" strategy is advantageous. In one of the early simulation studies about the effect of tool axis, i.e. lead and tilt angles, on process mechanics and dynamics Ozturk et al. [25] showed that the preferable tool axis depends on the cutting depth and step over. They studied the conditions to avoid tool tip contact and the effect of lead and tilt angles on cutting forces form error and chatter stability through process simulations. In a recent study, Yigit and Lazoglu [26] studied the effect of lead and tilt angles in 5-axis ball-end milling of flexible parts. They considered cutting force, torque, part vibration and surface quality to propose an effective strategy for tool axis selection. The simulation results are validated by machining experiments. In all of these studies, it is emphasized that there are preferable tool axis vectors for improved process mechanics and dynamics depending on the cutting conditions such as step over and cutting depth. However, as the cutting conditions vary along 5-axis milling cycles, the preferable tool axis vectors also vary and needs to be identified at several cutter locations along the tool path. Process simulation can be performed along the tool path, i.e. at the cutter locations given in the CLS file, in order to obtain variation of cutting forces, torque, tool deflection or stability limits. However, modification of tool axis at the corresponding CL points based on the simulation results requires the machined surface location and surface normal vector to be known at these CL points. This is one of the main limitations to automatic tool axis modification considering process mechanics and dynamics in simultaneous 5-axis milling.

Considering that the surface normal information is not explicitly given in the CLS file, there is a need for an approach to extract the surface data directly from the CLS file so that automatic tool path modification based on process simulations can be possible without any need to iterative tool path generation at the CAM side. There have been a couple of studies to extract the machined surface information from CLS file. Ozturk and Lazoglu [2] used CL points to approximate the machined surface. The first derivative of the machined surface at the corresponding CL point is approximated by forward difference between two consecutive CL points. However, CL points diverse from the CC points with increased lead and tilt angles. Thus, such an approach may lead to significant deviations when the tool has spatial motion especially in 5-axis milling. Later, Tunc and Budak [27] proposed an approach to relate the surface normal information to the $\mathrm{CL}$ points based on generation of a reference tool path file with zero lead and tilt angle to have the tool axis coincident with the surface normal. Then, the surface normal vectors were matched to the CL points in the original tool path file. This approach enabled extraction of cutting conditions and surface information directly from CLS file; however, the need for generation of a reference file was the drawback as it might not be practical for all cases to generate a CLS with zero lead and tilt angle.

In this paper, a practical and novel approach is proposed to retrieve surface data, i.e. surface location points and surface normal vector, directly from an already generated CLS file. As the surface retrieval is based on the generated CL file, the possible tangent and curvature discontinuities on the surface are filtered out at a rate close to the tool path generation density. The novelty and practicality of the proposed approach is its operability on a single CLS file so that the tool path modification in 5axis free-form surface ball-end milling becomes possible and very easy without any need for extra information or tool path generation at the CAM side. Henceforth, the paper is organized as follows: 5-axis ball-end milling geometry is presented in Section 2, which is followed by the fundamentals of tool path generation in order to define the relation between surface normal, tool axis, lead angle, tilt angle, $C C$ point and $C L$ point. The 
proposed approach to retrieve surface normal and $\mathrm{CC}$ points from the generated CL file is given in Section 4. The verification of the proposed approach is presented in Section 5 based on comparison of the retrieved surface information from the CLS file with the CAD data, where offline tool path compensation is also demonstrated based on known tool deflections for a 5-axis milling cycle. The paper is finalized with conclusions.

\section{5-Axis milling process geometry}

Tool path generation involves geometrical transformations from the machined surface to the cutting tool. In this section, 5 -axis milling process geometry is briefly described together with the coordinate systems involved. In 5-axis milling, there are three coordinate systems to define tool location and orientation with respect to the workpiece, i.e. workpiece coordinate system (WCS), process coordinate system (FCN) and tool coordinate system (TCS) as illustrated in Fig. 1.

WCS is constructed by the global axis defining the orientation of the workpiece in three-dimensional space. The process coordinate system is formed by the feed (F), cross-feed (C) and surface normal $(\mathrm{N})$ vectors relating the tool motion to the workpiece surface. Finally, the tool axis coordinate system is formed by the tool axis vector (ta) and two transversal axis, i.e. $x$ and $y$, to the tool axis. It is noteworthy to state that the tool axis is a rotated vector around $\mathrm{F}$ and $\mathrm{C}$ vectors with respect to the surface normal, N. Such a rotational transformation relates TCS to FCN. In other words, the tool axis vector is defined with respect to the surface normal vector by two concatenated rotations, firstly by amount of lead angle around cross-feed vector and then by amount of tilt angle around feed vector.

\section{Tool path computation in 5-axis milling}

In 5-axis milling tool path generation, initially, the CC points are identified from the CAD model of the surface

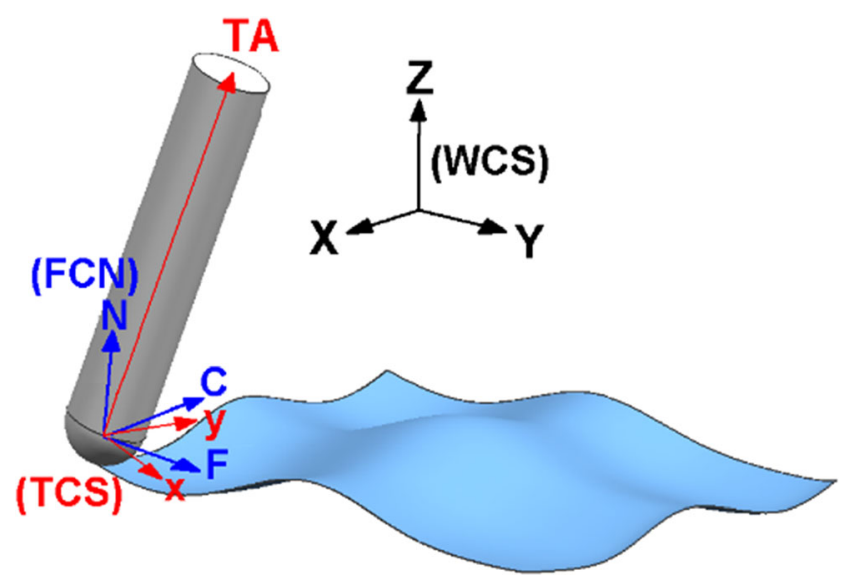

Fig. 1 Coordinate systems used for tool path generation in 5-axis milling according to the intended point density and surface stock [18] as illustrated in Fig. 2a (see black points). The surface, i.e. CC point, information is accompanied with the surface normal vector at each $\mathrm{CC}$ point to calculate the tool axis vector with known lead and tilt angles. Finally, the CL point is calculated as a vector summation of surface normal and tool axis vector from $\mathrm{CC}$ point to $\mathrm{CL}$ point (see Fig. 2b). In this section, vector algebra needed for tool path generation in 5axis milling is presented to explain how the CL point, $\mathrm{CC}$ point, surface normal and tool axis are related to each other.

\subsection{Converting the surface information to CLS file}

The $p$ th cutter contact point, $\mathrm{CC}_{\mathrm{p}}$, and the corresponding surface normal vector, $n_{\mathrm{p}}$, are used to calculate the spherical centre of the cutting tool, i.e. point $S_{p}$.

$S_{p}=\mathbf{C C}_{p}+\mathbf{R n}_{p}$

Then, the tool axis needs to be calculated to obtain CL point, which is done by sequentially rotating the surface normal vector around feed and cross-feed vectors by tilt angle and lead angle, respectively [18]. Thus, the rotation needs the feed and cross-feed vectors to be known, where the feed vector is calculated between two consecutive CC points.

$f_{p}=\mathrm{CC}_{p+1}-\mathrm{CC}_{p}$

Considering that the feed vector, the surface normal vector and the cross-feed vector form an orthogonal basis, the crossfeed vector is the cross-multiplication from the surface normal vector to the feed vector.

$c_{p}=n_{p} \times f_{p}$

After establishing the feed vector using the consecutive cutter contact points and the cross-feed vector using the surface normal and feed vectors, the concatenation of the two rotations applies on the surface normal vector to calculate the tool axis vector at the $p$ th $C C$ point, i.e. $t a_{p}$. The generalized rotation matrices around these two vectors are written as follows:

$\boldsymbol{t a}_{\boldsymbol{p}}=\left[R\left(\boldsymbol{c}_{\boldsymbol{p}}\right.\right.$, lead $\left.)\right]\left[R\left(\boldsymbol{f}_{\boldsymbol{p}}\right.\right.$, tilt $\left.)\right] \boldsymbol{n}_{\boldsymbol{p}}$

where $[R(u, \theta)]$ is the generalized rotation matrix around vector $u$ by angle of $\theta$

$R(u, \theta)=\left[\begin{array}{ccc}u_{i}^{2}+\left(1-u_{i}^{2}\right) C_{\theta} & u_{i} u_{j} \mathrm{~V}_{\theta}+u_{z} S_{\theta} & u_{i} u_{k} \mathrm{~V}_{\theta}-u_{j} S_{\theta} \\ u_{i} u_{j} \mathrm{~V}_{\theta}-u_{z} S_{\theta} & u_{j}^{2}+\left(1-u_{j}^{2}\right) C_{\theta} & u_{j} u_{k} \mathrm{~V}_{\theta}+u_{i} S_{\theta} \\ u_{i} u_{k} \mathrm{~V}_{\theta}+u_{j} S_{\theta} & u_{j} u_{k} \mathrm{~V}_{\theta}-u_{i} S_{\theta} & u_{k}^{2}+\left(1-u_{k}^{2}\right) C_{\theta}\end{array}\right]$ 
Fig. 2 Tool path generation in 5axis milling. a CC points. $\mathbf{b}$ Tool axis calculation. $\mathbf{c} C L$ points and $\mathrm{CC}$ points

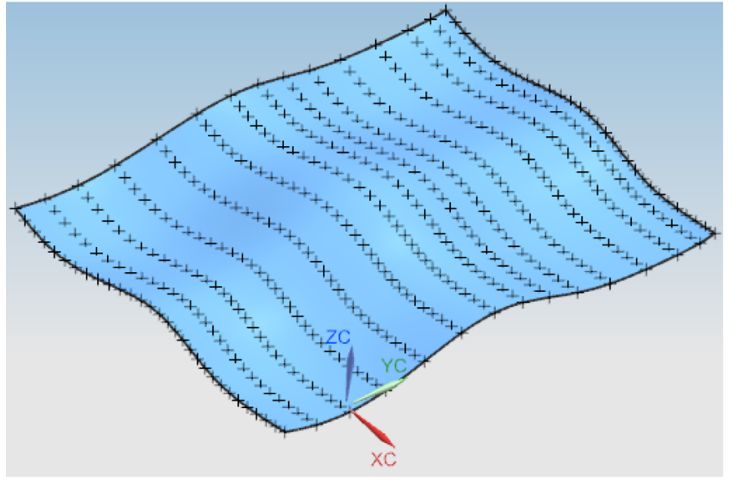

(a) CC points

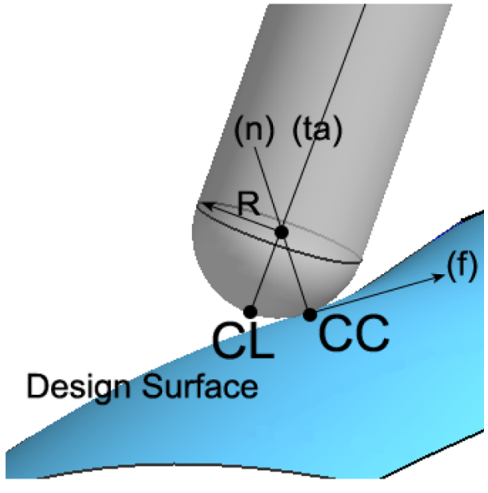

(b) Tool axis calculation

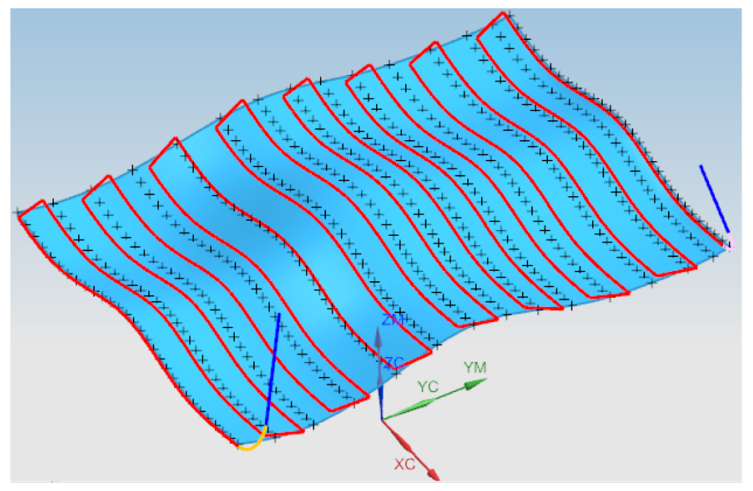

(c) CL points and CC points

$$
C_{\theta} \cos (\theta), S_{\theta} \sin (\theta), V_{\theta} 1-\cos (\theta) \text { and } \boldsymbol{u}=\left[\begin{array}{l}
\boldsymbol{u}_{\boldsymbol{i}} \\
\boldsymbol{u}_{\boldsymbol{j}} \\
\boldsymbol{u}_{\boldsymbol{k}}
\end{array}\right]
$$

Finally, the $p$ th cutter location point, CLp, is obtained using the corresponding tool axis vector and the spherical centre of the cutting tool. In other words, the CC point is translated along the resultant vector of surface normal and tool axis vectors multiplied by spherical radius of the tool.

$$
\mathbf{C L}_{p}=S_{p}-\mathbf{R t a}_{p}=\mathbf{C C}_{p}+R\left(n_{p}-\mathbf{t a}_{p}\right)
$$

Then, the calculated cutter location points and the corresponding tool axis vectors are exported in the CLS file format as shown in Fig. 3. It should be noted again that the $C L$ point coordinates and the tool axis unit vector are the only geometrical information contained in the CLS file. In other words, the CC point and the surface normal vector information are transformed to $\mathrm{CL}$ point and tool axis vector information throughout the geometrical transformation given from Eqs. 1 to 5. As either CC point, surface normal vector or cross-feed vector is not provided in the CLS file; the machined surface information cannot be retrieved directly from the CLS file, when required.
Having fundamental tool path generation technique in 5-axis milling is presented in this section, the proposed approach for extraction of the machined surface information using the information given in the CLS file is detailed in the next section.

\section{Extraction of surface data from CLS file}

In tool path modification, the surface normal and the $\mathrm{CC}$ point should be known as emphasized in the previous section. For instance, in order to compensate the tool deflection the tool tip location, i.e. CL point, must be offset along the surface normal direction. However, the CLS file does not include this information. If this information cannot be extracted from the CLS file, tool path modification will require a new tool path to be generated at the CAM side, where even local tool path modification may not be possible. This recursive relation blocks automatic tool path modification. Hence, it is required to retrieve the surface information directly from the CL file, which is described in this section. The purpose of the proposed approach is extracting the design surface data from the CL file for practical use of the simulation results on tool path modification. 
Fig. 3 Representative CLS file

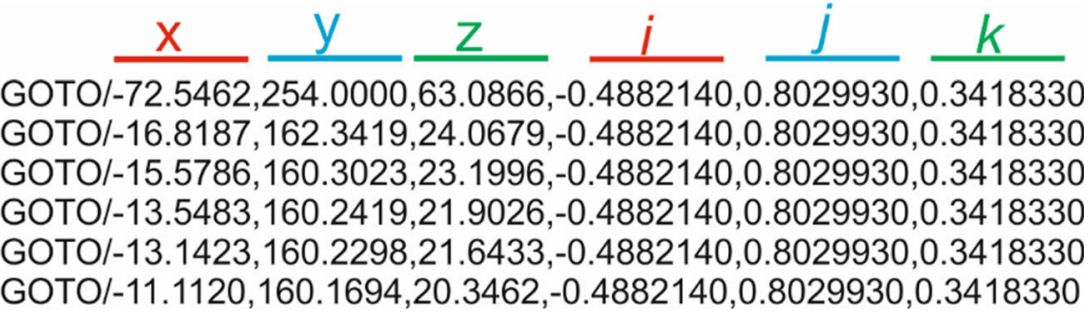

\subsection{Calculation of surface normal and cutter contact point}

The estimation of surface normal vector through the CLS file requires the feed and cross-feed vectors to be calculated. Two consecutive points along the tool path are required in order to calculate the unit feed vector, f. However, only the CL points are provided in the CLS file and using CL points to calculate the unit feed vector leads to significant errors as illustrated in Fig. 4, where the same tool path portion with different lead and tilt angle combinations are shown. Considering the tool path generation approach, it can be said that at any CC point, the coordinates of the $\mathrm{CL}$ point depend on the tool axis and hence the lead and tilt angles with respect to the machined surface.

In Fig. $4, f_{1}$ and $f_{3}$ are drawn between two consecutive CL points, whereas $f_{2}$ and $f_{4}$ are drawn between two consecutive CC points. It is seen that $f_{1}$ and $f_{3}$ differ from each other in terms of location and direction. However, $f_{2}$ and $f_{4}$ are the same as they are drawn between the CC points, which are absolute surface locations. This is due to the fact that for a known CC point, the CL point coordinates vary with lead and tilt angles. Thus, the CL point in the form given by the CLS file is useless to calculate the unit feed vector. However, this can be tackled by calculating the unit feed vector between two consecutive spherical centre points. The beauty of using the spherical centre of the tool is that it does not vary with lead and tilt angles as the cutting tool is rotated around the spherical centre to avoid gouging. In other words, the $\mathrm{CC}$ points and spherical centre of the tool are the absolute points generated for the surface to be machined, whereas the CL points are relative to tool axis so that in 5-axis milling, the vector from the $\mathrm{CC}$ point to the spherical centre corresponds to the surface normal vector and the vector from the CL point to the spherical centre corresponds to the tool axis vector.

The spherical centre of the tool can be calculated for known combination of CL point and tool axis vector by re-arranging Eq. 5:

$S_{p}=\mathbf{C L}_{p}+\mathbf{R t a}_{p}$

Then, the unit feed vector at the $p$ th cutter location is written as follows:

$f_{p}=\frac{S_{p+1}-S_{p}}{\left|S_{p+1}-S_{p}\right|}$

Similarly, the cross-feed vector is established through two closest spherical centre points as illustrated in Fig. 5.

In Fig. 5, $S_{\mathrm{p}}$ and $S_{\mathrm{r}}$ are the spherical centres corresponding to $p$ th and $r$ th CL points holding the below condition:

$\boldsymbol{r}=\operatorname{argmin}_{i}\left\{\left|\boldsymbol{S}_{\boldsymbol{p}}-\boldsymbol{S}_{i}\right|\right\}$

The unit cross-feed vector at the $p$ th cutter location is written as follows:

$c_{p}=\frac{S_{r}-S_{p}}{\left|S_{r}-S_{p}\right|}$

After obtaining the unit feed and cross-feed vectors, the surface normal at the $p$ th cutter location is obtained as crossproduct from cross-feed to the feed vector as illustrated in Fig. 5.

$n_{p}=c_{p} \times f_{p}$

Fig. 4 Construction of unit-feed vector

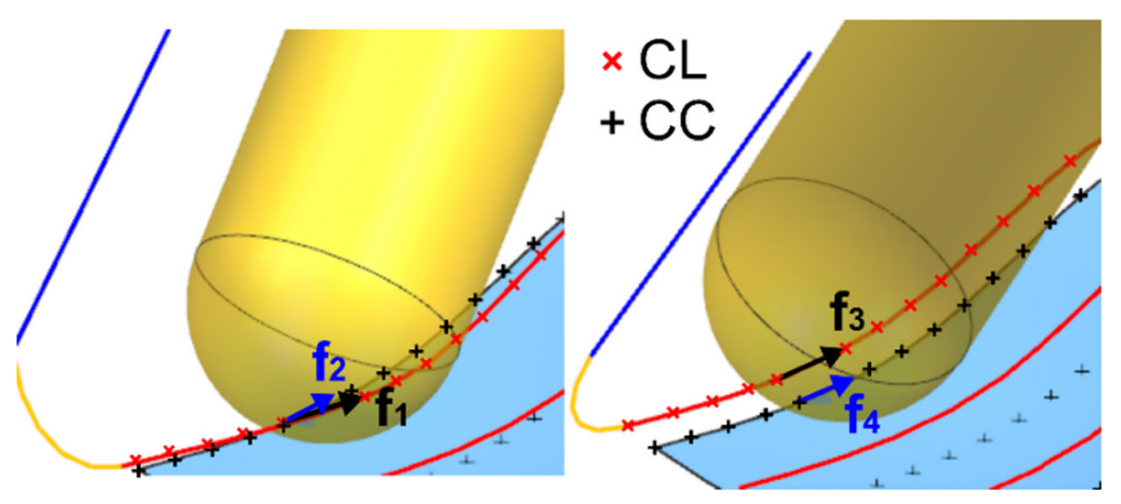


Fig. 5 Extraction of surface normal from CLS file. a Set of spherical centre points. b Construction of unit cross-feed vector

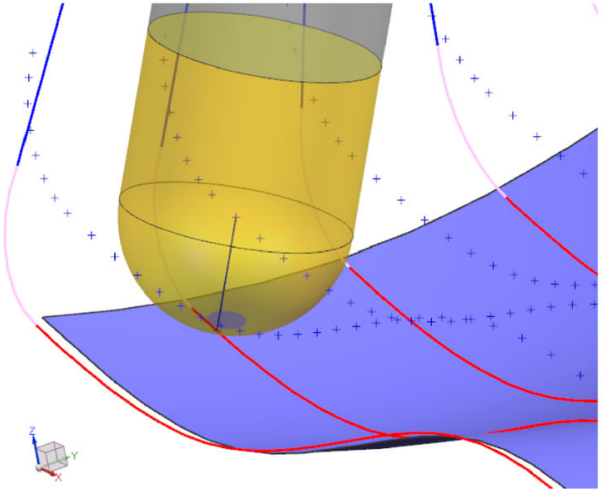

(a) Set of spherical centre points

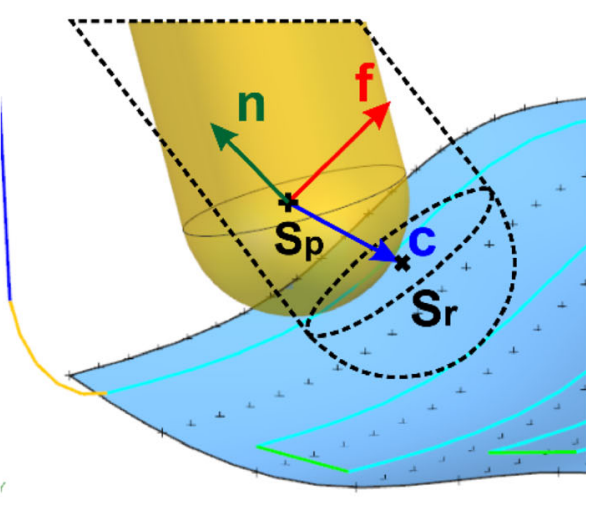

(b) Construction of unit cross feed vector
Once the surface normal vector corresponding to each cutter location point is obtained, it is used to calculate the cutter contact point by re-arranging Eq. 1 :

$$
\mathrm{CC}_{p}=S_{p}-\mathrm{Rn}_{p}
$$

In this section, the use of cutter location and tool axis vector information provided in the CLS file to retrieve the cutter contact points is explained. The calculated cutter contact points are then can be used to represent the machined surface for tool path modification so that the need for a new tool path generation at the CAM side is eliminated letting automatic tool path modification especially for tool deflection compensation or tool axis optimization.

\section{Verification of the surface extraction method}

The proposed approach for extraction of the design surface information from the CLS file is verified through representative case studies as given in this section. As the purpose of the paper is extracting the design surface data from the tool path, the experimental verification is based on comparison of the extracted surface information with the CAD data in terms of surface location points and surface normal vectors. The case studies are selected considering the surface types machined by 5-axis milling. In the first case, a free-form surface (see Fig. 6a), which is flexible in $\mathrm{u}$ and $\mathrm{v}$ directions, is considered for different tool path generation point density settings whereas in the second case, finishing of a rotor blade (see Fig. 6b) is considered.

The verification is performed in terms of the surface location error and the angular deviation of the surface normal vector, which are calculated by face-to-face deviation checking. For such a purpose, a new surface is fitted through the retrieved surface location points. Then, face-to-face deviation checking analysis is performed at number of points between the original CAD model and the new fitted surface as shown in see Fig. 6. In Section 5.3, the use of retrieved surface information for tool path compensation purpose is demonstrated, where it is assumed that the surface location error is known from CMM (coordinate measurement machine) measurements.

\subsection{Case 1: free-form surface milling}

Isometric free-form surfaces are widely designed and machined in several industries such as automotive, aerospace and die-mold. Thus, verification of the proposed approach is of great importance to show its applicability in wide variety of industries. In this case, a free-form isometric surface shown in Fig. $6 \mathrm{a}$ is considered under different tool path generation
Fig. 6 Illustration of face-to-face deviation checking. a Case 1. b Case 2

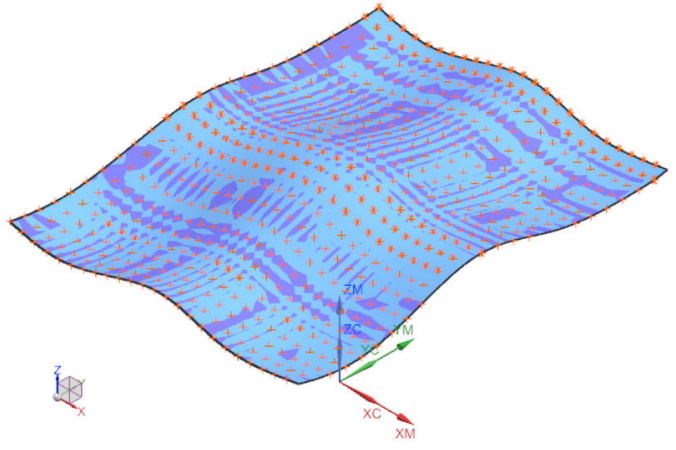

(a) Case 1

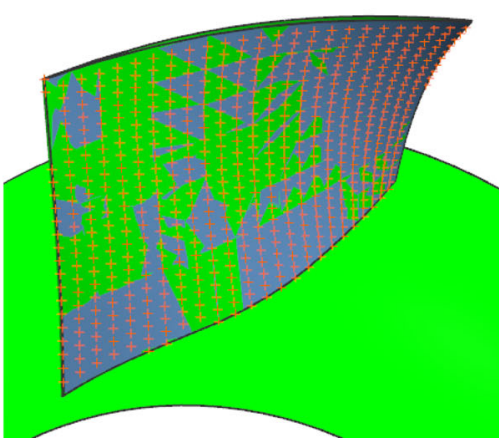

(b) Case 2 
Table 1 Tool path generation parameters for case 1

\begin{tabular}{lllll}
\hline & Path 1 & Path 2 & Path 3 & Path 4 \\
\hline Number of cutting steps & 15 & 15 & 50 & 50 \\
Point density per step & 40 & 40 & 50 & 100 \\
Lead/tilt angle $\left({ }^{\circ}\right)$ & $10 / 10$ & $25 /-30$ & $10 / 10$ & $10 / 10$ \\
\hline
\end{tabular}

parameters to represent different machining stages such as roughing and finishing. In general, the fundamental differences between the roughing and finishing passes are the cutter location density and the surface stock.

The surface with basic dimensions of $65 \times 65 \mathrm{~mm}$ in $\mathrm{xy}$ plane, is flexible in $\mathrm{u}$ and $\mathrm{v}$ directions, and samples both concave and convex regions with varying curvature. The tool path generation parameters such as the number of cutting steps, point density for each cutting step, and lead and tilt angles are presented in Table 1. In tool path generation, 8-mmdiameter ball-end mill tool is used, where a representative tool path is shown in Fig. 7.

The effect of the programmed lead and tilt angles on the accuracy of the results is assessed through comparison of the results obtained from path 1 and path 2 . The variation of accuracy with the number of cutting steps can be observed by comparing path 1 and path 3. Finally, the effect of point density on accuracy of the proposed approach is evaluated by comparing path 3 and path 4 . In Fig. 8, the accuracy results are given, where the distance deviation and angular deviation of the surface normal vector are plotted.

In Fig. 8a, c, the accuracy obtained by path 1 and path 2 are compared. It is observed that accuracy of the proposed approach is independent of the programmed lead and tilt angle, i.e. tool axis setting. This is due to the fact that the feed and cross-vectors are calculated based on the spherical centre of the ball-end tool, which does not change with tool axis vector as emphasized in the previous section. In this case, the maximum surface location error reaches up to $40 \mu \mathrm{m}$, whereas the angular deviation of the surface normal vector is $0.6^{\circ}$. Considering that the tool path is a representative roughing

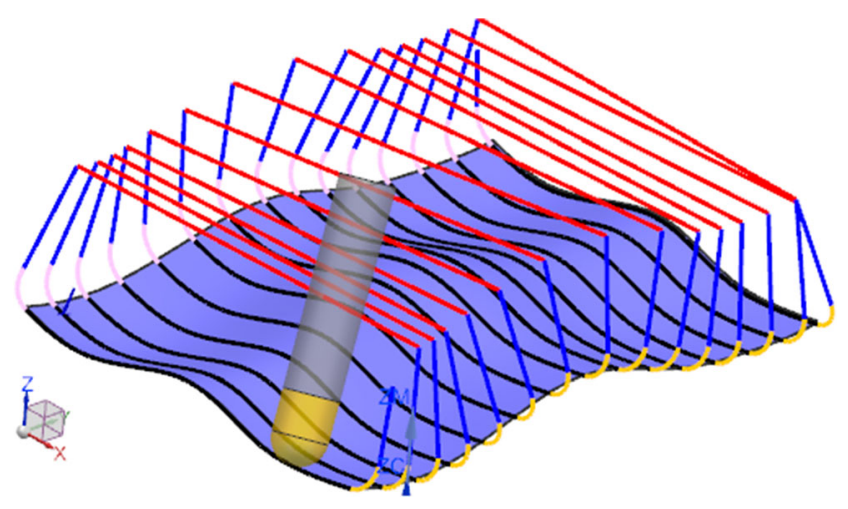

Fig. 7 Representative tool path (path 1) pass, it can be said that the obtained accuracy of the retrieved surface data is reasonable.

In Fig. $8 b$, d, the accuracy results for path 3 and path 4 are plotted. In path 3 , the maximum surface location error drops down to $7 \mu \mathrm{m}$, which was $40 \mu \mathrm{m}$ for path 1 and path 2 . Similarly, the maximum angular deviation of the surface normal vector substantially decreases to $0.07^{\circ}$, which was $0.7^{\circ}$ for path 1 and path 2 . A similar observation can be made as the accuracy on path 3 and path 4 is compared, where the number of points per step is increased from 50 to 100 . Thus, it can be concluded that the proposed approach performs even more accurately as the point density increases, which is more likely in finishing passes. The summary of the results is given in Table 2 .

\subsection{Case 2: blade milling}

Rotor blades are one of the general application of isometric free-form surfaces and 5-axis milling in aerospace and power generation industries. In case 2, the proposed approach is applied on a finishing tool path, which is generated for blade milling. The surface location points and surface normal error are calculated using the proposed approach and the results are compared through face-to-face checking analysis. The finishing pass is generated for side $\mathrm{A}$ and side $\mathrm{B}$ of the blade. In the generated tool path, there are 51 cutting steps each of which has 100 points, where the tool is $12-\mathrm{mm}$-diameter ballend mill.

The surface location error along the tool path is shown in Fig. 9a, where it is seen that the maximum surface location error is $0.4 \mu \mathrm{m}$ for side A and $0.8 \mu \mathrm{m}$ for side B. The surface normal angular deviation along the tool path is plotted in Fig. 9b. The maximum angular deviation is $0.2^{\circ}$ for side $\mathrm{A}$ and $0.3^{\circ}$ for side $\mathrm{B}$.

The results are summarized in Table 3, where the average surface location error is 0.05 and $0.008 \mu \mathrm{m}$ for side A and side $\mathrm{B}$, respectively. The average angular deviation on the surface normal is $0.011^{\circ}$ and $0.009^{\circ}$ for side $A$ and side $B$, respectively.

Considering the results achieved both in case 1 and case 2, it can be concluded that the proposed approach can accurately retrieve the machined surface information, i.e. surface location and normal, from the CLS file. It is observed that the accuracy of the results is not affected by the tool axis settings at all. The only critical parameter is the point density in the tool path, i.e. the number of cutting steps and the cutter location points per cutting step. It is seen that the accuracy increases with the point density, which means that the proposed approach can conform to the accuracy expected from the tool path either roughing or finishing so that the retrieved surface information can be used for modification of a tool path generated for 5-axis free-form surface machining. 
Fig. 8 Face-to-face deviation checking results in case 1 . a Effect of programmed lead/tilt angle. b Effect of point density. c Effect of programmed lead/tilt angle. $\mathbf{d}$ Effect of point density

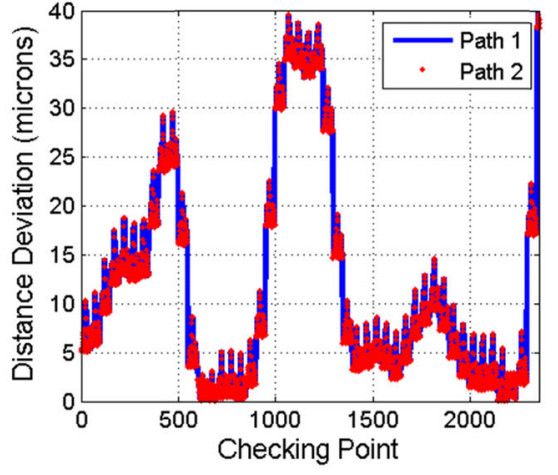

(a) Effect of programmed lead/tilt angle

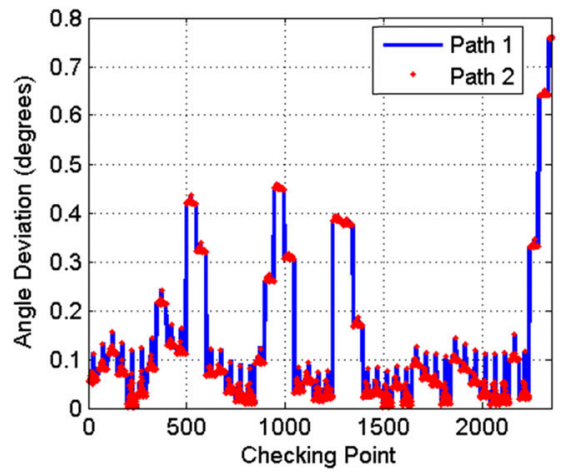

(c) Effect of programmmed lead//tilt angle

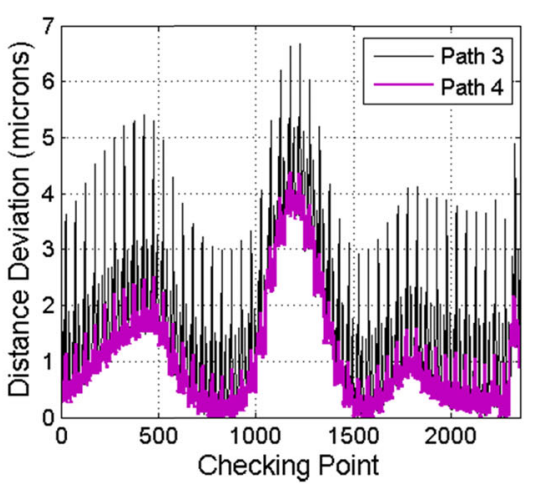

(b) Effect of point density

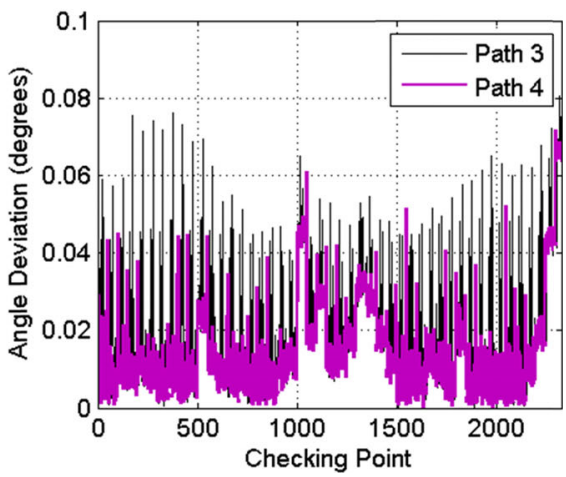

(d) Effect of point density

\subsection{Case 3: demonstration of tool path compensation in 5-axis milling}

Tool path compensation, either online or offline, is one of the widely used tool path modification techniques, where the form error might be caused by relative deflection between the tool and workpiece. In this section, the use of the proposed surface information extraction approach for tool path compensation purposes is demonstrated. In this representative case, the form error is accepted to be caused by only the cutting tool, which known through process simulation of the tool path shown in Fig. 7. The known form error information is then used to compensate the tool deflection by shifting the tool position along the surface normal vector at each cutter location point on the tool path.
The variation of the simulated form error at each cutter location point is plotted in Fig. 10a, where it is seen that the maximum and minimum form errors are around 20 and $2 \mu \mathrm{m}$. As the surface information is retrieved at each cutter location point, there is one-to-one match between the form error data and retrieved surface information. In order to compensate the tool deflection, the compensated cutter contact points are calculated by the shifting the original cutter contact point along the negative surface normal vector direction by amount of the form error.

$\mathrm{CC}_{p}^{\mathrm{comp}}=\mathrm{CC}_{\boldsymbol{p}}-\varepsilon_{p} \boldsymbol{n}_{p}$

where $\mathbf{C C}_{\boldsymbol{p}}^{\text {comp }}$ is the compensated cutter contact point and $\varepsilon_{p}$ is the form error corresponding to the $p$ th cutter location point.

Table 2 Face-to-face deviation checking summary for case 1

\begin{tabular}{llllllll}
\hline & $\begin{array}{l}\text { Number of } \\
\text { points checked }\end{array}$ & $\begin{array}{l}\text { Minimum } \\
\text { distance error (mm) }\end{array}$ & $\begin{array}{l}\text { Maximum } \\
\text { distance error (mm) }\end{array}$ & $\begin{array}{l}\text { Average } \\
\text { distance error (mm) }\end{array}$ & $\begin{array}{l}\text { Minimum } \\
\text { angle error (o) }\end{array}$ & $\begin{array}{l}\text { Maximum distance error (o) } \\
\text { distance error (o) }\end{array}$ \\
\hline Path 1 & 2486 & 0.00000 & 0.06143 & 0.01366 & 0.00103 & 0.76927 & 0.15513 \\
Path 2 & 2486 & 0.00001 & 0.06146 & 0.01366 & 0.00099 & 0.76922 & 0.15508 \\
Path 3 & 2499 & 0.00000 & 0.01337 & 0.00194 & 0.00029 & 0.07012 & 0.03452 \\
Path 4 & 2493 & 0.00000 & 0.00412 & 0.00183 & 0.00012 & 0.06034 & 0.02089 \\
\hline
\end{tabular}


Fig. 9 Face-to-face deviation checking results in case 2 . a Surface location error. b Surface normal angular deviation

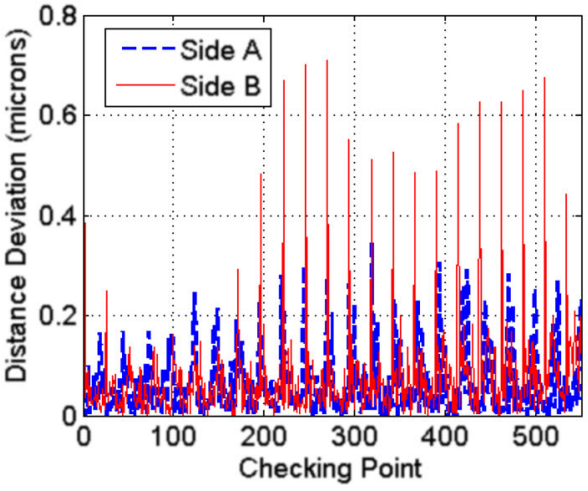

(a) Surface location error

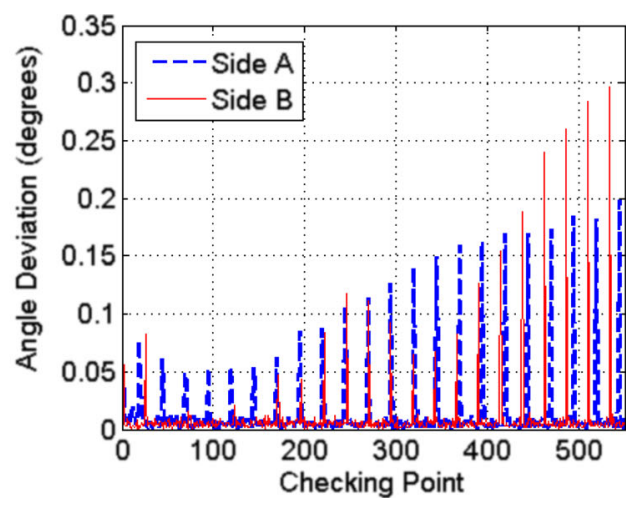

(b) Surface normal angular deviation

Table 3 Face-to-face deviation checking summary for case 2

\begin{tabular}{|c|c|c|c|c|c|c|c|}
\hline & $\begin{array}{l}\text { Number of } \\
\text { points checked }\end{array}$ & $\begin{array}{l}\text { Minimum } \\
\text { distance error (mm) }\end{array}$ & $\begin{array}{l}\text { Maximum } \\
\text { distance error }(\mathrm{mm})\end{array}$ & $\begin{array}{l}\text { Average } \\
\text { distance error }(\mathrm{mm})\end{array}$ & $\begin{array}{l}\text { Minimum } \\
\text { angle error (o) }\end{array}$ & $\begin{array}{l}\text { Maximum } \\
\text { distance error (o) }\end{array}$ & $\begin{array}{l}\text { Average } \\
\text { distance error (o) }\end{array}$ \\
\hline Side A & 550 & 0.00000 & 0.00030 & 0.00005 & 0.00030 & 0.29686 & 0.01138 \\
\hline Side B & 550 & 0.00000 & 0.00080 & 0.00008 & 0.00027 & 0.29599 & 0.00973 \\
\hline
\end{tabular}

Then, the compensated cutter location is computed using Eq. 5. Representative cutting steps of the compensated tool path are shown in Fig. 10b.

As the purpose of this example is demonstrating the use of the proposed approach in tool path modification, no real machining experiments have been performed, though it is shown that the surface information retrieved from the original tool path file can be easily integrated with the simulated tool deflection for tool path compensation. The same operation can be performed for tool axis optimization once the optimized tool axis vectors are available through process simulations.

\section{Conclusions}

The increased contouring capability, thanks to the ball-end and rotational axis of the cutting tool, complicates the process geometry and mechanics in 5-axis free-form surface machining. Process simulations can be used for selection of improved cutting conditions through simulation-based process optimization techniques. In order to make the most of the benefit from the simulation effort, the simulation results needs to be used for automatic tool path modification purposes. Most of the machining cycle simulation systems are based on tool path information provided in CLS file format, where simulation
Fig. 10 Tool path compensation based on simulated form error. a Form error, b Compensated tool path (XY view)

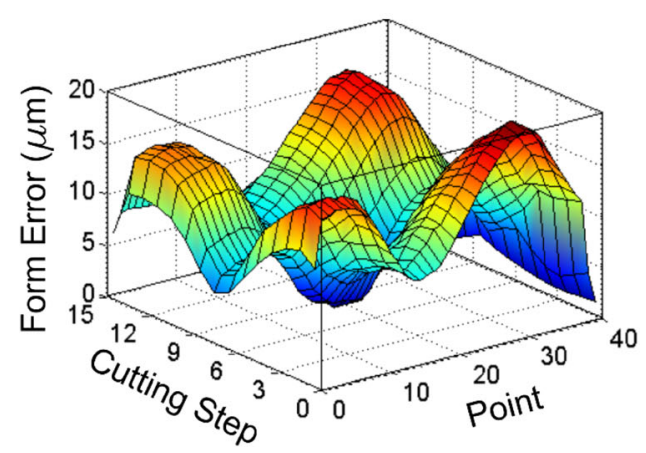

(a) Form error

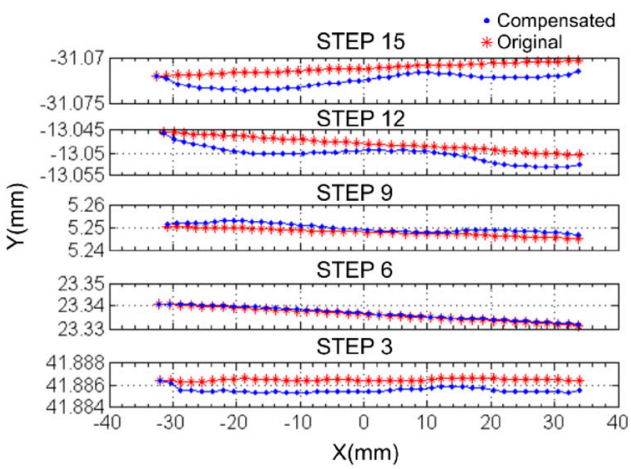

(b) Compensated tool path (XY view) 
results can be used to identify tool deflection or optimized tool axis vectors along a 5 -axis milling cycle. Automatic tool path modification requires the simulated tool deflection and or optimized tool axis vectors to be used for re-calculation of the tool path from the original tool path. However, this requires the machined surface information such as surface location or surface normal vector, which are not explicitly available in the tool path file. In this paper, a novel and practical approach is proposed in order to retrieve the machined surface information from a given CLS file, which is generated for 5-axis free-form surface machining. The proposed approach is based on inverse geometrical solution of the tool path information. The estimations are verified through face-to-face deviation checking between the original and the retrieved surface data. It is shown that the machined surface information can be retrieved accurate enough to re-calculate the tool path for modification purposes. The accuracy of the approach does not rely on the variation of the tool axis in the tool path file. It is seen that the maximum surface location error for roughing type of operations is around $40 \mu \mathrm{m}$, which decreases down to $4 \mu \mathrm{m}$ for finishing type of operations. The surface location error drops down below $1 \mu \mathrm{m}$ for smooth surfaces such as rotor blades. The maximum angular deviation between the retrieved surface normal vector and the original surface is around $0.8^{\circ}$ for roughing operations, which decreases down to $0.08^{\circ}$ for finishing operations. The use of the proposed approach in tool deflection compensation is demonstrated, and it is shown that the simulation data can be easily integrated with the retrieved surface data for tool path compensation purposes.

Acknowledgments The author acknowledges the support of Turkish National Science Foundation under grant no. 105M038.

\section{References}

1. Budak E, Ozturk E, Tunc LT (2009) Modeling and simulation of 5axis milling processes. CIRP Ann Manuf Technol 58(1):347-350

2. Ozturk B, Lazoglu I (2006) Machining of free-form surfaces. Part I: analytical chip load. Int J Mach Tools Manuf 46(7):728-735

3. Ozturk B, Lazoglu I, Erdim H (2006) Machining of free-form surfaces. Part II: calibration and forces. Int J Mach Tools Manuf 46(7): 736-746

4. Zhu R, Kapoor SG, DeVor RE (2001) Mechanistic modeling of the ball end milling process for multi-axis machining of free-form surfaces. J Manuf Sci Eng 123(3):369-379

5. Fussell BK, Jerard RB, Hemmett JG (2003) Modeling of cutting geometry and forces for 5-axis sculptured surface machining. Comput Aided Des 35(4):333-346

6. Kim GM, Kim BH, Chu CN (2003) Estimation of cutter deflection and form error in ball-end milling processes. Int $\mathrm{J}$ Mach Tools Manuf 43(9):917-924
7. Eksioglu C, Kilic ZM, Altintas Y (2012) Discrete-time prediction of chatter stability, cutting forces, and surface location errors in flexible milling systems. J Manuf Sci Eng 134(6):061006

8. Ozturk E, Budak E (2010) Dynamics and stability of five-axis ballend milling. J Manuf Sci Eng 132(2):021003

9. Wan M, Kilic ZM, Altintas Y (2015) Mechanics and dynamics of multifunctional tools. J Manuf Sci Eng 137(1):011019

10. Altintas Y, Kilic ZM (2013) Generalized dynamic model of metal cutting operations. CIRP Ann Manuf Technol 62(1):47-50

11. Altintas Y, Kersting P, Biermann D, Budak E, Denkena B, Lazoglu I (2014) Virtual process systems for part machining operations. CIRP Ann Manuf Technol 63(2):585-605

12. Ong TS, Hinds BK (2003) The application of tool deflection knowledge in process planning to meet geometric tolerances. Int J Mach Tools Manuf 43(7):731-737

13. Lo CC, Hsiao CY (1998) A method of tool path compensation for repeated machining process. Int J Mach Tools Manuf 38(3):205213

14. Dépincé P, Hascoët JY (2006) Active integration of tool deflection effects in end milling. Part 2. Compensation of tool deflection. Int J Mach Tools Manuf 46(9):945-956

15. Ratchev S, Liu S, Becker AA (2005) Error compensation strategy in milling flexible thin-wall parts. J Mater Process Technol 162:673681

16. Habibi M, Arezoo B, Nojedeh MV (2011) Tool deflection and geometrical error compensation by tool path modification. Int $\mathrm{J}$ Mach Tools Manuf 51(6):439-449

17. Rao VS, Rao PVM (2006) Tool deflection compensation in peripheral milling of curved geometries. Int J Mach Tools Manuf 46(15): 2036-2043

18. Choi BK (1991) Surface modelling for CAD/CAM. Elsevier, New York

19. Ho MC, Hwang YR, Hu CH (2003) Five-axis tool orientation smoothing using quaternion interpolation algorithm. Int J Mach Tools Manuf 43(12):1259-1267

20. Gray PJ, Ismail F, Bedi S (2004) Graphics-assisted rolling ball method for 5-axis surface machining. Comput Aided Des 36(7): 653-663

21. Jun CS, Cha K, Lee YS (2003) Optimizing tool orientations for 5axis machining by configuration-space search method. Comput Aided Des 35(6):549-566

22. Beudaert X, Pechard PY, Tournier C (2011) 5-Axis tool path smoothing based on drive constraints. Int J Mach Tools Manuf 51(12):958-965

23. Chen HP, Kuo HH, Tsay DM (2009) Removing tool marks of blade surfaces by smoothing five-axis point milling cutter paths. J Mater Process Technol 209(17):5810-5817

24. Lim TS, Lee CM, Kim SW, Lee DW (2002) Evaluation of cutter orientations in 5-axis high speed milling of turbine blade. J Mater Process Technol 130:401-406

25. Ozturk E, Tunc LT, Budak E (2009) Investigation of lead and tilt angle effects in 5-axis ball-end milling processes. Int J Mach Tools Manuf 49(14):1053-1062

26. Yigit IE, Lazoglu I (2015) Analysis of tool orientation for 5-axis ball-end milling of flexible parts. CIRP Ann Manuf Technol

27. Tunc LT, Budak E (2009) Extraction of 5-axis milling conditions from CAM data for process simulation. Int J Adv Manuf Technol 43(5-6):538-550 